\title{
Effect of parentage misidentification on estimates of genetic parameters for milk yield in the Mediterranean Italian buffalo population
}

\author{
E. Parlato ${ }^{*}{ }^{1}$ and L. D. Van Vleck* $¥$ \\ *Department of Animal Science, University of Nebraska, Lincoln 68583-0908 \\ †Italian Buffalo Breeders' Association, via C. Battisti, 68, 81100, Caserta, Italy \\ fUSDA, Agricultural Research Service (ARS), Roman L. Hruska US Meat Animal Research Center, Lincoln, NE 68583-0908²
}

\section{ABSTRACT}

The objective of this study was to evaluate the effect of parentage misidentification on estimation of genetic parameters for the Italian buffalo population for milk yield from 45,194 lactation records of 23,104 Italian buffalo cows. Animals were grouped into 10 data sets in which sires and dams were DNA identified, or reported from the pedigree, or unknown. A derivativefree restricted maximum likelihood method was used to estimate components of variance with a repeatability model. The model contained age at calving nested within parity and days from calving to conception as linear covariates, herd-year-seasons as fixed effects, and additive genetic, permanent environmental, and temporary environmental effects as random effects. Estimates of heritability $( \pm \mathrm{SE})$ ranged from $0.00 \pm 0.099$ (sires and dams as reported in the pedigree) to $0.39 \pm$ 0.094 (sires DNA identified and dams as reported in the pedigree). When identification of sires was as reported in the pedigree, estimates of heritability were close to zero. These small estimates indicate that a large proportion of reported paternity is incorrect. When sires are unknown and dams are DNA identified, the proportion of variance due to sires seems to be captured in the estimate of permanent environmental variance as a fraction of phenotypic variance. Therefore, as heritability decreased, permanent environmental variance increased about the same amount. Data sets with dams identified from pedigree and sires DNA identified showed the largest estimate of heritability (0.39), which was essentially the same as when dams were DNA identified (0.38). This result supports that most dams are correctly reported from the pedigree. Genetic progress should be much greater with bulls DNA identified because of greater heritability, but without artificial insemination and progeny testing, progress would be slow

Received August 22, 2011

Accepted February 20, 2012 .

${ }^{1}$ Corresponding author: e.parlato@anasb.it

${ }^{2} \mathrm{USDA}$ is an equal opportunity provider and employer. and would depend mostly on selection of sires based on dam estimated breeding values. Implementation of artificial insemination programs and DNA testing to identify sires are the keys for increasing genetic progress in the Italian buffalo population.

Key words: dairy buffalo, pedigree misidentification, heritability

\section{INTRODUCTION}

Genetic evaluations of buffalo cows and bulls by BLUP methods assume that all known genetic relationships among individuals included in the analysis are correct. However, in several studies of cattle (Christensen et al., 1982; Geldermann et al., 1986), between 4 and $23 \%$ of paternal identifications were found to be false. In the Italian buffalo population, rates of sire and dam misidentification were found to be $24 \%$ and $20 \%$ [Italian Buffalo Breeders' Association (ANASB), Caserta, Italy; E. Parlato, , 2010).

Pedigree errors are expected to bias estimation of genetic parameters (Van Vleck, 1970a), breeding values of individuals (Van Vleck, 1970b; Israel and Weller, 2000; Banos et al., 2001), correct ranking of tested bulls in progeny testing, and expected genetic progress (Van Vleck, 1970b; Geldermann et al., 1986; Israel and Weller, 2000). As would be expected, bias increases as the proportion of records with errors increases (Van Vleck, 1970a). Ignoring existing relationships also results in decreased estimates of genetic variance (Dong et al., 1988). Thus, higher estimates of heritability might result if more precise pedigree data were used. Visscher and Thompson (1992) suggested that some differences in estimates of genetic parameters may be due to the type of relatives contributing to parameter estimates. They estimated variance components for fat yield using an animal model, a model with only female relationships, and a sire model. They found a decrease in fat yield heritability when sires were ignored.

Estimates of variance components for yield traits are required by the Italian Buffalo Breeders' Association for national genetic evaluation of the Mediterranean 
Italian buffalo population. Rosati and Van Vleck (2002) reported heritability estimates for 270-d lactation yields of milk, fat, protein, and Mozzarella cheese, and for percentages of fat and protein of $0.14,0.11,0.14,0.13$, 0.17 , and 0.10 . In their study, 10,663 lactation records of 3,873 individuals were analyzed.

Natural mating is the system applied by most Italian buffalo enterprises. Breeding is, generally, carried out by group mating (2 bulls with 25 buffalos) and calving takes place on open ranges. Under these conditions, paternity is difficult to establish. Thus, to avoid pedigree errors due to sire misidentification, only sires identified by DNA testing are included in the relationship matrix for the genetic evaluation. Sires in the reported pedigree are classified as unknown.

Recently, use of DNA markers has provided a more accurate method of identifying individuals and verifying parentage. For the buffalo population, the DNA markers of choice in parental testing are microsatellites (Heyen et al., 1997) that are codominant (Fries, 1993). Essentially, typing of several microsatellites is carried out for an offspring and its alleged parent. A sire or a dam is eliminated as a parent when the genotype of the offspring is not compatible with the parental genotype for at least 1 microsatellite. As the probability of exclusion is the probability of rejecting an alleged parent that is a random individual within the population, the probability of exclusion depends on the marker type, number of alleles, and allelic frequencies in the population to be used for paternity testing.

The objective of this study was to investigate the effect of parentage misidentification on estimates of variance components and genetic parameters for milk yield for the Mediterranean Italian buffalo population.

\section{MATERIALS AND METHODS}

\section{Data}

Data obtained from the ANASB database included historical yield and pedigree information for buffalo enrolled in ANASB programs throughout Italy. All records were extended to $270 \mathrm{~d}$ for lactations less than 270 d. The complete data set included records from first to fifth lactations of all buffalo born after 1980 . Editing consisted of accepting only lactation records of buffalo having more than 150 or less than $570 \mathrm{~d}$ of lactation and having, in the current lactation, calvingto-conception intervals (days open) between 20 and 730 d. Parities were grouped into 3 different classes: parities 1,2 , and $\geq 3$. Ninety-three age classes were formed. Twenty-one classes were assigned to parity 1 (age at calving from 25 to $45 \mathrm{mo}$ ), 29 classes were assigned to parity 2 (age at calving from 37 to $65 \mathrm{mo}$ ), and
43 classes were assigned to parities $\geq 3$ (age at calving from 45 to 90 mo or greater). All age classes contained a single age in months, with the exception that the last class in parities $\geq 3$ included all ages $>90$ mo. Days open (DO) in the current lactation was approximated by taking the difference between 2 consecutive calving intervals and subtracting the length of the average buffalo gestation period $(310 \mathrm{~d})$. For first lactations, an average DO of $170 \mathrm{~d}$ was assigned. Lactation records were grouped into $7 \mathrm{DO}$ classes; DO were rounded to the nearest month to form classes corresponding to mo 1 to 6 . Months $\geq 7$ constituted the last class. Contemporary groups were formed by grouping records of buffalo calving in the same herd in the same year and season of calving within the year. Three seasons of calving were defined: January to April, May to August, and September to December. The data set was reduced by removing records in contemporary groups with $<5$ observations.

To evaluate the effect of parentage misidentification on estimates of variance components and genetic parameters, lactation records were grouped into 2 data sets: 1) only dams identified by DNA testing were included (DS1) and 2) only dams identified from the pedigree were included (DS2). Within each data set, 4 subsets were formed by changing the type of sire identification: a) DNA tested (DS1a and DS2a), b) from the pedigree or unknown (DS1b and DS2b), c) only sires unknown (DS2c and DS2c), and d) only sires from the pedigree (DS1d and DS2d). In Table 1, the total number of buffalo and the number of sires and dams with different identifications are summarized for each data set.

The first data set, DS1, consisted of 15,702 lactation records of 10,608 buffalo with all dams identified by DNA testing and with sires or identified by DNA testing, or unknown sire, or sire identified from the pedigree. The DS1 was divided into 4 subsets: DS1a consisted of 6,351 lactation records of 5,846 buffalo with sires identified by DNA testing, DS1b consisted of 9,351 lactation records of 4,762 buffalo with sires unknown or identified from the pedigree, DS1c consisted of 6,697 lactation records of 3,584 buffalo with unknown sires, and DS1d consisted of 2,654 lactation records of 1,178 buffalo with sires only identified from the pedigree.

The second data set, DS2, consisted of 29,492 lactation records of 12,496 buffalo with all dams identified from the pedigree and with sires or identified by DNA testing or identified from the pedigree or unknown. The DS2 was divided into 4 subsets: DS2a consisted of 3,511 lactation records of 2,074 buffalo with sires identified by DNA testing, DS2b consisted of 25,981 lactation records of 10,422 buffalo with unknown sires or with 
Table 1. Total number of buffalo (n), number of buffalo with sire identified by DNA testing (DNA), with unknown sire (NO), and with sire identified from the pedigree $(\mathrm{P})$; with dam identified by DNA testing (DNA) and with dam identified from the pedigree (A) for 10 data sets

\begin{tabular}{|c|c|c|c|c|c|c|}
\hline \multirow[b]{2}{*}{ Data set ${ }^{1}$} & \multirow[b]{2}{*}{$\mathrm{n}$} & \multicolumn{3}{|c|}{ Sire } & \multicolumn{2}{|c|}{ Dam } \\
\hline & & DNA & $\mathrm{NO}$ & $\mathrm{P}$ & DNA & $\mathrm{A}$ \\
\hline DS1 & 10,608 & 5.846 & 3.584 & 1,178 & 10,608 & - \\
\hline DS1a & 5,846 & 5,846 & - & - & 5,846 & - \\
\hline DS1b & 4,762 & - & 3,584 & 1,178 & 4,762 & - \\
\hline DS1c & 3,584 & - & 3,584 & - & 3,584 & - \\
\hline DS1d & 1,178 & - & - & 1,178 & 1,178 & - \\
\hline DS2 & 12,496 & 2,074 & 6,211 & 4,211 & - & 12,496 \\
\hline DS2a & 2,074 & 2,074 & - & - & - & 2,074 \\
\hline DS2b & 10,422 & - & 6.211 & 4.211 & - & 10,422 \\
\hline DS2c & 6,211 & - & 6,211 & - & - & 6,211 \\
\hline DS2d & 4,211 & - & - & 4.211 & - & 4,211 \\
\hline
\end{tabular}

${ }^{1}$ DS1 = only dams identified by DNA testing were included; DS1a = DS1 subset in which sire identification was by DNA testing; DS1b = DS1 subset in which sire identification was from the pedigree or unknown; DS1c = DS1 subset in which only sire identification was unknown; DS1d = DS1 subset in which sire identification was only from the pedigree; DS2 = only dams identified from the pedigree were included; DS2a = DS2 subset in which sire identification was by DNA testing; DS2b = DS2 subset in which sire identification was from the pedigree or unknown; DS2c = DS2 subset in which only sire identification was unknown; DS2d = DS2 subset in which sire identification was only from the pedigree.

sires identified from the pedigree, DS2c consisted of 14,984 lactation records of 6,211 buffalo with unknown sires, and DS2d consisted of 10,997 lactation records of 4,211 buffalo with sires identified from the pedigree. The number of different sires and dams for each data set is reported in Table 2. In DS1, 1,041 sires and 7,381 dams were identified by DNA testing and 453 sires were identified from the pedigree, whereas in DS2, 437 sires were identified by DNA testing, 972 sires were identified from the pedigree, and 10,125 dams were identified from the pedigree.

\section{Statistical Analyses}

Estimates of variance components for milk yield were obtained with the following mixed model:

$$
y_{h i j k l}=H Y S_{h}+L C_{i}+D O_{j}+a_{k}+c_{k}+e_{h i j k l}
$$

where $y_{\text {hijkl }}$ is lactation yield $(\mathrm{kg})$ of milk with twice daily milking and lactation length of $270 \mathrm{~d}, H Y S_{h}$ is the effect of herd-year-season class $h, L C_{i}$ is the effect of age at calving-parity class $i, D O_{j}$ is the effect of

Table 2. Total number of buffalo (N), number of sires identified by DNA testing (DNA), sires identified from the pedigree (P), dams identified by DNA testing (DNA), and dams identified from the pedigree (A) included in 10 data sets

\begin{tabular}{|c|c|c|c|c|c|}
\hline \multirow[b]{2}{*}{ Data set ${ }^{1}$} & \multirow[b]{2}{*}{$\mathrm{N}$} & \multicolumn{2}{|c|}{ Sire } & \multicolumn{2}{|c|}{ Dam } \\
\hline & & DNA & $\mathrm{P}$ & DNA & $\mathrm{A}$ \\
\hline DS1 & 10,608 & 1,041 & 453 & 7,381 & - \\
\hline DS1a & 5,846 & 1,041 & - & 4,506 & - \\
\hline DS1b & 4,762 & - & 453 & 3,680 & - \\
\hline DS1c & 3,584 & - & - & 3,035 & - \\
\hline DS1d & 1,178 & - & 453 & 988 & - \\
\hline DS2 & 12,496 & 437 & 972 & - & 10,125 \\
\hline DS2a & 2,074 & 437 & - & - & 1,933 \\
\hline DS2b & 10,422 & 0 & 972 & - & 8,560 \\
\hline DS2c & 6,211 & 0 & - & - & 5,271 \\
\hline DS2d & 4,211 & 0 & 972 & - & 3,664 \\
\hline
\end{tabular}

${ }^{1}$ DS1 = only dams identified by DNA testing were included; DS1a = DS1 subset in which sire identification was by DNA testing; DS1b = DS1 subset in which sire identification was from the pedigree or unknown; DS1c = DS1 subset in which only sire identification was unknown; DS1d = DS1 subset in which sire identification was only from the pedigree; DS2 = only dams identified from the pedigree were included; DS2a = DS2 subset in which sire identification was by DNA testing; DS2b = DS2 subset in which sire identification was from the pedigree or unknown; DS2c = DS2 subset in which only sire identification was unknown; DS2d = DS2 subset in which sire identification was only from the pedigree. 
calving-to-conception class $j, a_{k}$ is the additive genetic effect of animal $k, c_{k}$ is the permanent environmental effect associated with buffalo $k$, and $e_{h i j k l}$ is the random residual effect associated with the record. Additive genetic, permanent environmental, and residual effects were assumed to be mutually uncorrelated.

Variance components and fractions of phenotypic variance were estimated from single-trait analyses using a derivative-free algorithm (Boldman et al., 1995) to obtain estimates that maximize the residual (restricted) likelihood (REML). The stopping criteria was set at $1 \times 10^{-6}$ for the variance of -2 (log-likelihood) in the simplex. The number of rounds allowed was up to 250 before restarting. Local convergence was declared when the variance of the simplex was less than $10^{-6}$, after which restarts were made until convergence at global maximum was declared when $-2(\log$-likelihood $)$ did not change to the second decimal.

\section{RESULTS AND DISCUSSION}

The number of lactation records, unadjusted means, and standard deviations for milk yield $(\mathrm{kg})$ for 10 data sets are shown in Table 3. Among the 10 data sets, lactation milk yield averaged between 2,489 $\pm 532 \mathrm{~kg}$ (DS2c) and 2,654 $\pm 596 \mathrm{~kg}$ (DS1d). The average milk production for all data sets was greater than that reported in Rosati and Van Vleck (2002; 2,286 $\pm 492 \mathrm{~kg})$.

Estimates of variance components and genetic parameters for 10 data sets are presented in Table 4. Data sets DS1 and DS2 differ by the method of identification of dams to use in the relationship matrix (dams identified by DNA testing for DS1, and dams identified from the reported pedigree for DS2). Estimates of heritabil- ity were greatly different across data sets, ranging from $0.00 \pm 0.099$ (DS1d) to $0.39 \pm 0.094$ (DS2a). Because the 2 data sets differ only by identification of dams, the decrease in estimate of heritability from DS1 to DS2 might be attributed to the much larger proportion of records with dams identified from pedigree in DS2 (29,492 vs. 15,702$)$.

Among the data sets of DS1, the largest estimate (0.38) of heritability occurred when sires and dams were identified by DNA testing (DS1a). Estimates of heritability decreased when records of animals with unknown sires (DS1c, $\left.\mathrm{h}^{2}=0.28\right)$ and sires identified from the pedigree (DS1b, $\left.\mathrm{h}^{2}=0.25\right)$ were added to DS1a.

When identification of sires was as reported in the pedigree, estimates of heritability were close to zero for both subsets $(0.07$ and 0.00 , for DS2d and DS1d, respectively). These small estimates indicate that a large proportion of reported paternity is incorrect (Van Vleck, 1970b; Geldermann et al., 1986; Dong et al., 1988). If information of sires from the pedigree were correct (DS1d and DS2d), the estimate of heritability would be expected to be greater than the estimate of heritability when sires are unknown (DS1c and DS2c). Sire misidentification from the reported pedigree appears to cause the estimate of the animal genetic variance to go to zero (Van Vleck, 1970b; Geldermann et al., 1986).

When sires are unknown (DS1c), the proportion of variance due to sires seems to be captured in the estimate of permanent environmental variance as a fraction of phenotypic variance $\left(c^{2}\right)$. Therefore, as heritability decreased, permanent environmental variance increased about the same amount. Thus, repeatability $\left(\mathrm{h}^{2}+\mathrm{c}^{2}\right)$ did not vary much across data sets.

Table 3. Number of lactation records $(\mathrm{N})$, unadjusted means, and standard deviations for yield of milk $(\mathrm{kg})$ for 10 data sets [with sire/dam identified by DNA testing (DNA), unknown sire (NO), sire identified from the pedigree $(\mathrm{P})$, and dam identified from the pedigree $(\mathrm{A})$ ]

\begin{tabular}{lllrrr}
\hline & & & & Unadjusted \\
Data set $^{1}$ & Sire & Dam & $\mathrm{N}$ & SD \\
\hline DS1 & DNA, NO, P & DNA & 15,702 & 2,570 & 558 \\
DS1a & DNA & DNA & 6,351 & 2,560 & 553 \\
DS1b & NO, P & DNA & 9,351 & 2,576 & 561 \\
DS1c & NO & DNA & 6,697 & 2,548 & 543 \\
DS1d & P & DNA & 2,654 & 2,654 & 596 \\
DS2 & DNA, NO, P & A & 29,492 & 2,530 & 548 \\
DS2a & DNA & A & 3,511 & 2,493 & 542 \\
DS2b & NO, P & A & 25,981 & 2,535 & 549 \\
DS2c & NO & A & 14,984 & 2,489 & 532 \\
DS2d & P & A & 10,997 & 2,603 & 565 \\
\hline
\end{tabular}

${ }^{1}$ DS1 = only dams identified by DNA testing were included; DS1a = DS1 subset in which sire identification was by DNA testing; DS1b = DS1 subset in which sire identification was from the pedigree or unknown; DS1c = DS1 subset in which only sire identification was unknown; DS1d = DS1 subset in which sire identification was only from the pedigree; DS2 = only dams identified from the pedigree were included; DS2a $=$ DS2 subset in which sire identification was by DNA testing; DS2b = DS2 subset in which sire identification was from the pedigree or unknown; DS2c = DS2 subset in which only sire identification was unknown; DS2d = DS2 subset in which sire identification was only from the pedigree. 
Table 4. Estimates of variance components, heritability $\left(\mathrm{h}^{2}\right)$, environmental effects (SE in parentheses), and repeatability (rep) for yield of milk $(\mathrm{kg})$ for 10 data sets (with sire/dam identified by DNA testing (DNA); unknown sire (NO); sire identified from the pedigree (P); dam identified from the pedigree $(\mathrm{A})]$

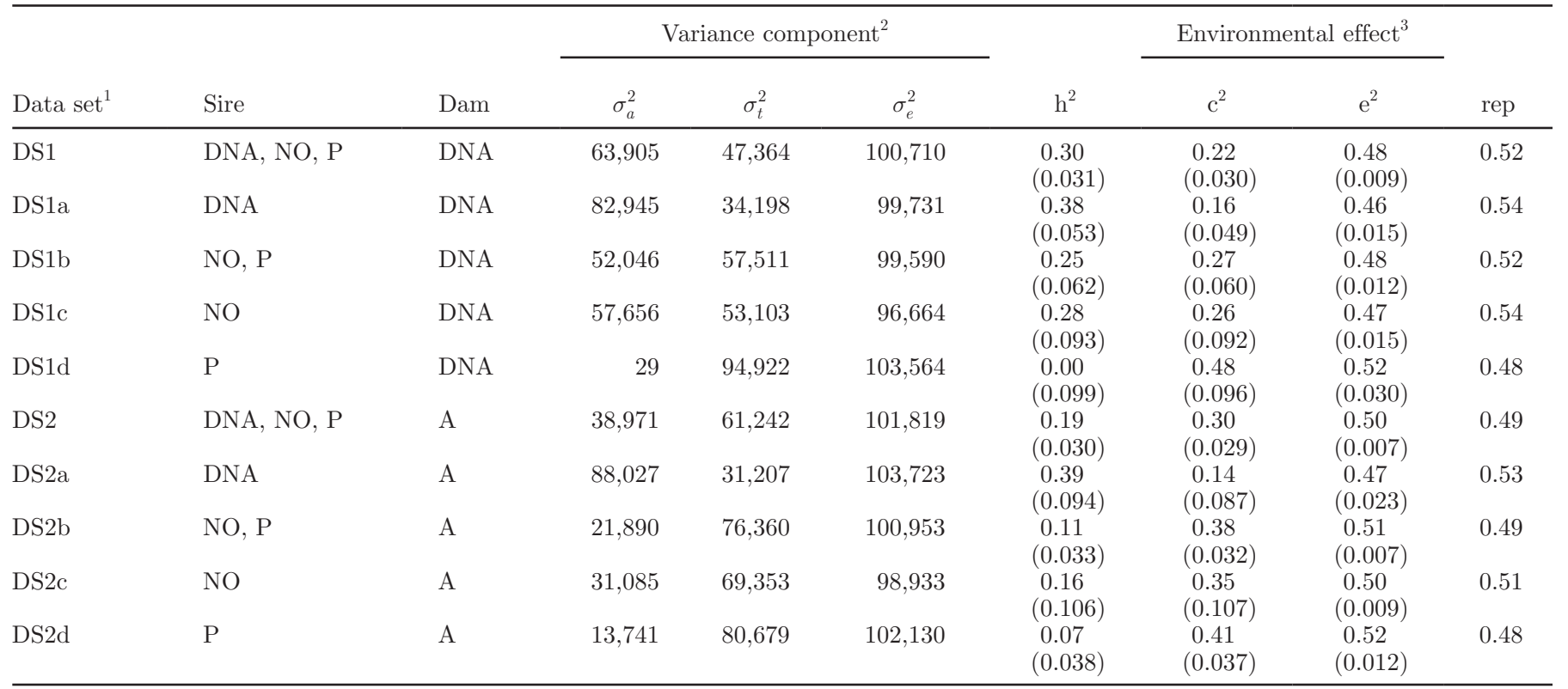

${ }^{1}$ DS1 = only dams identified by DNA testing were included; DS1a = DS1 subset in which sire identification was by DNA testing; DS1b = DS1 subset in which sire identification was from the pedigree or unknown; DS1c = DS1 subset in which only sire identification was unknown; DS1d = DS1 subset in which sire identification was only from the pedigree; DS2 = only dams identified from the pedigree were included; DS2a = DS2 subset in which sire identification was by DNA testing; DS2b = DS2 subset in which sire identification was from the pedigree or unknown; DS2c $=$ DS2 subset in which only sire identification was unknown; DS2d = DS2 subset in which sire identification was only from the pedigree. ${ }^{2} \sigma_{a}^{2}=$ estimated variance component for additive genetic effects; $\sigma_{t}^{2}=$ estimated variance component for permanent environmental effects; $\sigma_{e}^{2}=$ estimated error variance.

${ }^{3} \mathrm{c}^{2}=$ permanent environmental effect; $\mathrm{e}^{2}=$ temporary environmental effect.

Among the subsets of DS2 (dams identified from the reported pedigree), DS2a (sires identified by DNA testing) had the largest estimate of heritability (0.39), which was essentially the same as when dams were identified by DNA testing (DS1a). This result suggests that dams are mostly correctly reported from the pedigree. As with DS1, estimates of heritability for data sets of DS2 decreased with apparent incorrect reporting of sire identification. Estimates of permanent environmental variance and residual variance also increased as the estimate of heritability decreased.

Estimates of heritability from DS2b (0.16) and DS2c (0.11) were comparable to the estimate of 0.14 obtained by Rosati and Van Vleck (2002). In their study, lactation records of animals with sires and dams reported from the pedigree were used. Therefore, the structure of the relationship matrix was more similar to that of DS2b and DS2c, in which all animals had dams as reported from the pedigree, with sires unknown and identified from the pedigree (DS2b) or with only unknown sires (DS2c). The DS1d and DS2d subsets had estimates of heritability close to zero. Estimates of heritability from the other subsets were greater than that obtained by Rosati and Van Vleck (2002).
The estimates of heritability from this study are evidence that a large proportion of sires reported from the pedigree are identified incorrectly. Therefore, the current requirement for genetic evaluation to use only records of buffalo with sires identified by DNA testing is supported. If sires are identified as reported in the pedigree, they would be reclassified as unknown. The effective heritability might be smaller (Van Vleck, 1970b; Geldermann et al., 1986; Dong et al., 1988), but more buffalo would be evaluated. If sires identified by DNA testing and sires identified from the pedigree were in the same herd, the contemporary groups would be larger. Larger contemporary groups in herds would lead to more accurate sire evaluation (Van Vleck, 1987), but with the assumption of correct identification. The results suggest that dams do not need to be identified by DNA testing. However, those data sets were relatively small.

The decreases in genetic gain tended to be greater for lower heritability traits and for higher levels of pedigree errors (Van Vleck, 1970a; Geldermann et al., 1986; Israel and Weller, 2000). Therefore, including only bulls identified by DNA testing in the pedigree for the genetic evaluation will lead to a much greater genetic progress. 
Moreover, without AI and progeny testing, progress would be slow and would depend mostly on selection of sires based on dam EBV. Even with DNA testing of sires, the sires may be gone before they have enough progeny to affect their evaluation. Those progeny would help in identifying the best bull-dams (paternal half sibs). Therefore, implementation of AI programs and DNA testing of sires are the keys to increasing genetic progress in the Italian buffalo population.

\section{REFERENCES}

Banos, G., G. R. Wiggans, and R. L. Powell. 2001. Impact of paternity errors in cow identification on genetic evaluations and international comparisons. J. Dairy Sci. 84:2523-2529.

Boldman, K. G., L. A. Kriese, L. D. Van Vleck, C. P. Van Tassell, and S. D. Kachman. 1995. A Manual for Use of MTDFREML. A Set of Programs to Obtain Estimates of Variances and Covariances. Agric. Res. Serv., US Department of Agriculture, Washington, DC.

Christensen, L. G., P. Madsen, and J. Petersen. 1982. The influence of incorrect sire-identification on the estimates of genetic parameters and breeding values. Pages 200-208 in Proc. 2nd World Congr. Genet. Appl. Livest. Prod. Editorial Garsi, Madrid, Spain.

Dong, M. C., L. D. Van Vleck, and G. R. Wiggans. 1988. Effect of relationships on estimation of variance components with an animal model and restricted maximum likelihood. J. Dairy Sci. 71:30473052 .

Fries, R. 1993. Mapping the bovine genome: Methodological aspects and strategy. Anim. Genet. 24:111-116.

Geldermann, H., U. Pieper, and W. E. Weber. 1986. Effect of misidentification on the estimation of breeding value and heritability in cattle. J. Anim. Sci. 63:1759-1768.

Heyen, D. W., J. E. Beever, Y. Da, R. E. Evert, C. Green, S. R. Bates, J. S. Ziegle, and H. A. Lewin. 1997. Exclusion probabilities of 22 bovine microsatellite markers in fluorescent multiplexes for semiautomated parentage testing. Anim. Genet. 28:21-27.

Israel, C., and J. I. Weller. 2000. Effect of misidentification on genetic gain and estimation of breeding values in dairy cattle populations. J. Dairy Sci. 83:181-187.

Rosati, A., and L. D. Van Vleck. 2002. Estimation of genetic parameters for milk, fat, protein and Mozzarella cheese yield in the Italian river buffalo population. Livest. Prod. Sci. 74:185-190.

Van Vleck, L. D. 1970a. Misidentification in estimating the paternal sib correlation. J. Dairy Sci. 53:1469-1474.

Van Vleck, L. D. 1970b. Misidentification and sire evaluation. J. Dairy Sci. 53:1697-1702.

Van Vleck, L. D. 1987. Contemporary groups for genetic evaluation. J. Dairy Sci. 70:2456-2464.

Visscher, P. M., and R. Thompson. 1992. Comparison between genetic variances estimated from different types of relatives in dairy cattle. Anim. Prod. 55:315-320. 